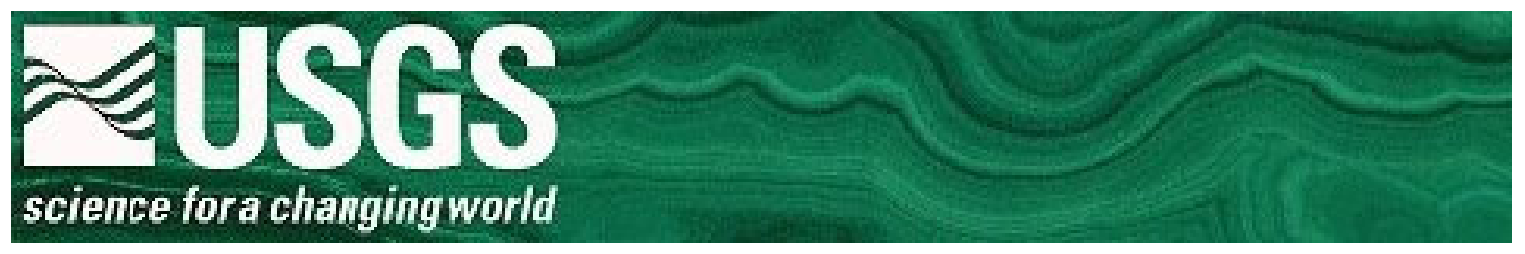

U.S. Geological Survey

Open-File Report 03-364

Version 1.0

\title{
Cx-02 Program Workshop on Modeling Complex Systems
}

By

\section{Victor G. Mossotti, Jo Ann Barragan, and Todd D. Westergard}

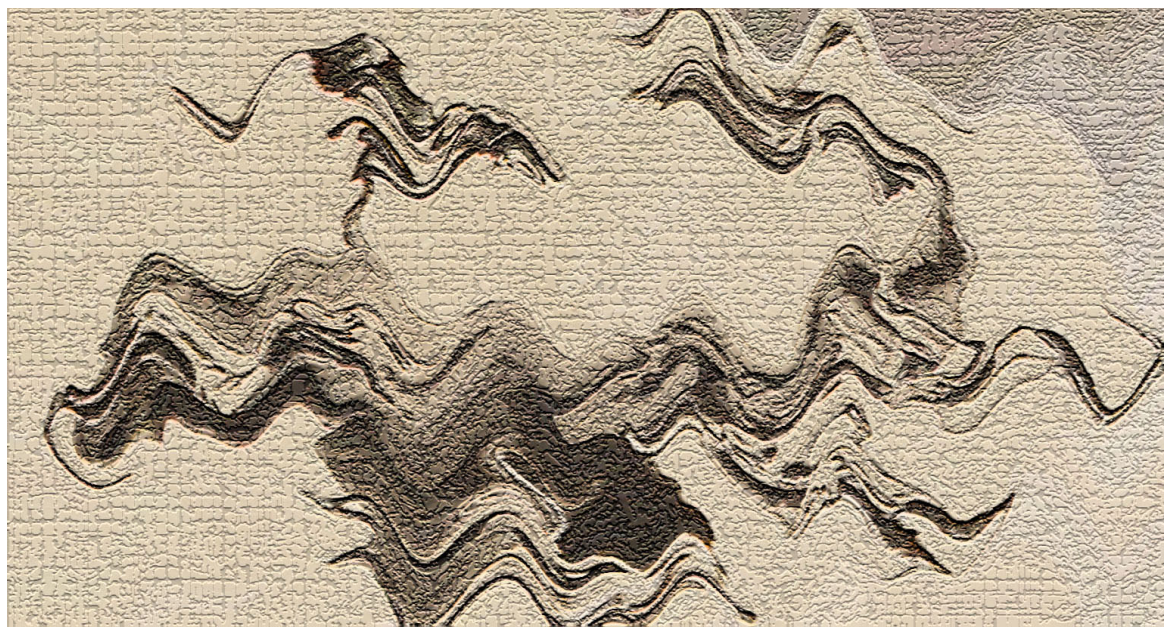

Image used for cover page on $\sim 25 \%$ of programs

\section{INTRODUCTION}

As chairman of the workshop on modeling complex systems, I would like to welcome the workshop participants and thank our sponsors, contributors, and steering committee for their support. I am especially grateful to Kathleen Johnson, USGS Mineral Resources Program Coordinator, for providing the primary financial support 
for this workshop and for her pioneering spirit in initiating the Complex Systems project in the Western Region Mineral Resources Team.

Complex systems are ubiquitous within the realm of the earth sciences. Geological systems consist of a multiplicity of linked components with nested feedback loops; the dynamics of these systems are non-linear, iterative, multi-scale, and operate far from equilibrium. That notwithstanding, I have noticed that, with the exception of papers on seismic studies, geology and geophysics work has been disproportionally underrepresented at regional and national meetings on complex systems relative to papers in the life sciences. This is something of a puzzle to me because geologists and geophysicists are, in many ways, preadapted to thinking of complex system mechanisms. Geologists and geophysicists think about processes involving large volumes of rock below the sunlit surface of Earth, the accumulated consequence of processes extending hundreds of millions of years in the past. Not only do geologists think in the abstract by virtue of the vast time spans, most of the evidence is out-ofsight.

A primary goal of this workshop is to begin to bridge the gap between the Earth sciences and life sciences through demonstration of the universality of complex systems science, both philosophically and in model structures.

Victor G. Mossotti Chairman 


\title{
CONTENTS
}

\section{Program}

\author{
Sponsors \\ Steering Committee \\ Short course outline \\ Schedule of presentations \\ List of presentations
}

\begin{abstract}
s
Workshop participants

GIS map

Participant data (in root)

.dbf file

Microsoft Excell .csv file

File Maker Pro .fp5 file

ESRI ArcView/Arc Map shape files (in folderin root)
\end{abstract}




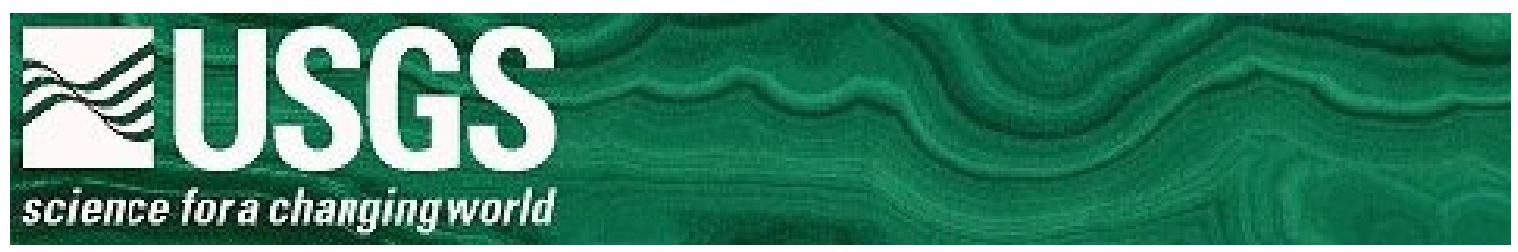

\section{Modeling Complex Systems Program and Abstracts}

\section{Short Course: November 19,2002 Workshop: November 20-21}

REID Engineering Laboratory Conference Facility, University of Nevada, Reno, Nevada

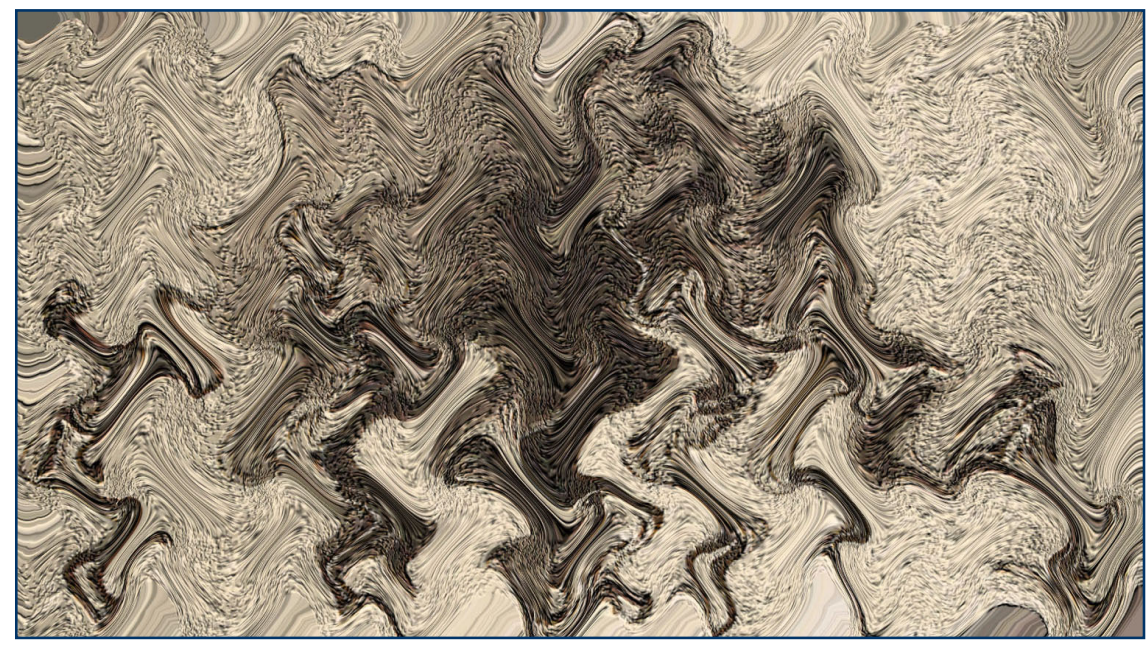

"These blind men, every one honest in his contentions and certain of having the truth, formed schools and sects and factions ..."

Buddha

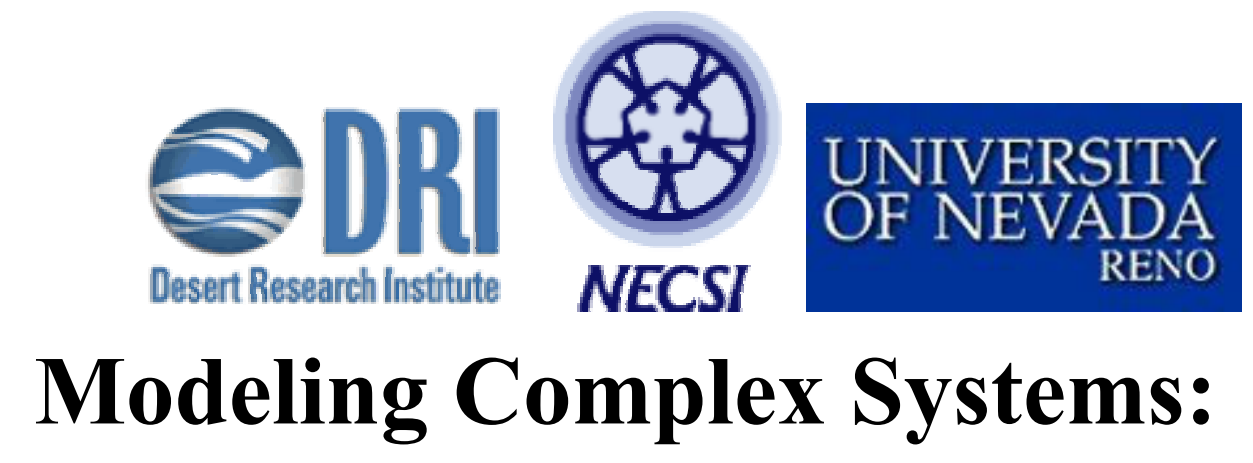




\title{
Workshop
}

\author{
November $20 \&$ 21, 2002 \\ Reid Engineering Laboratory Conference Center, \\ University of Nevada, Reno Nevada
}

\section{Sponsored by:}

\author{
Mineral Resources Program, U.S. Geological Survey \\ Desert Research Institute, Reno, Nevada \\ University of Nevada-Reno, College of Engineering \\ New England Complex Systems Institute, Cambridge, MA.
}

\section{Steering Committee:}

Victor G. Mossotti (Chairman), U.S. Geological Survey (650) 329-5284

Christopher C. Barton, U.S. Geological Survey, St. Petersburg, Florida

Jacoby Carter,

Yaneer Bar-Yam,

Wolfgang Bein,

Ahmad M. Itani,

Zann Gill,

Lui Lam,

U.S. Geological Survey, Lafayette, Louisiana

New England Complex Systems Institute, Cambridge

University of Nevada, Las Vegas

University of Nevada, Reno

MetaVu NetWork, Los Altos, CA

Sushil Louis,

W. Alan McKay,

San Jose State University

University of Nevada, Reno

Desert Research Institute, Reno

Kelly T. Redmond,

Desert Research Institute, Reno

Bernard Zygelman,

University of Nevada, Las Vegas

Ellen Jacobson (Local Arrangements), Univ. of Nevada, Reno

JoAnn Barragan (Manuscript Coordination), U.S. Geological Survey 


\section{Modeling Complex Systems The Short Course}

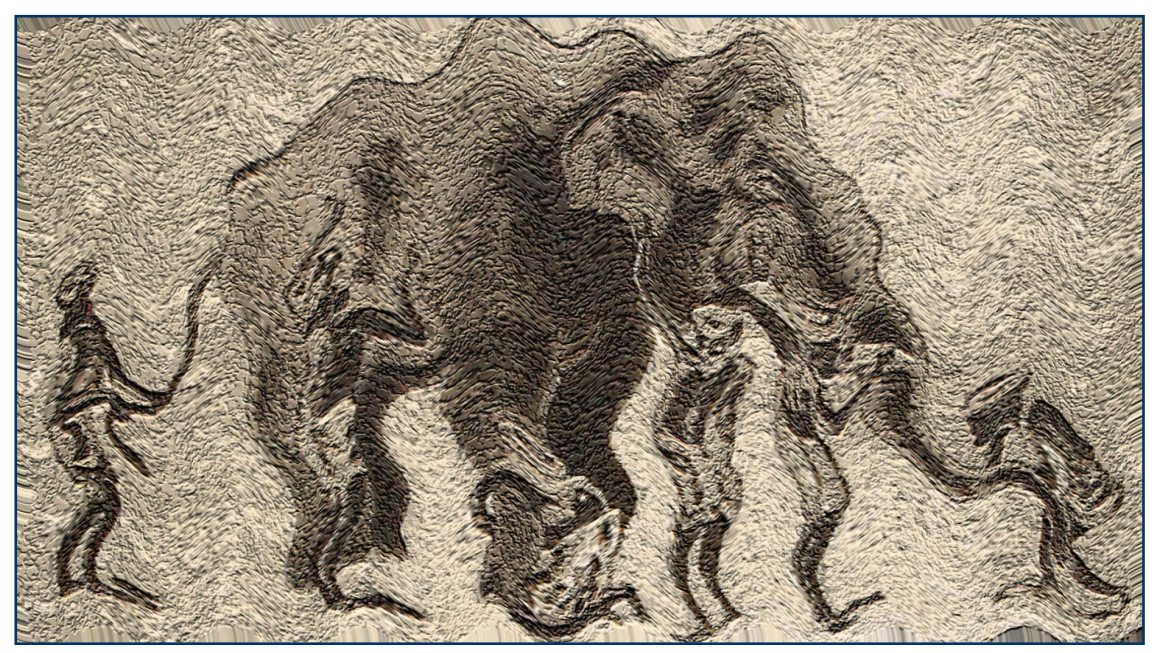

Day: $\quad$ Wednesday, November 19, 2002

Location: Harry Reid Laboratory Conference Center University of Nevada at Reno (see map above)

Hours: $\quad$ 8:00 Continental Breakfast; Registration 8:00 - 9:00 (For lunch, we suggest the buffet at the University Inn) 9:00am-5:00pm

Instructor: Yaneer Bar-Yam

This course will introduce several approaches to the study of complex systems, and will give an overview of the application of complex systems science to research and modeling. Basic concepts will be reviewed and implications for the study of inanimate, biological, sociological and engineered systems will be discussed. 


\section{Workshop Program \\ Nov 20-21, 2002 \\ Reid Engineering Laboratory Conference Facility \\ University of Nevada, Reno}

Day One: Wednesday Morning

8:00 Continental Breakfast \& Registration

9:00 Welcoming comments:

Ted Batchman, Dean, School of Engineering, UNR

Peter Vikre, Team Chief Scientist-WR, MRT, USGS

Victor Mossotti, USGS, Workshop Chairman

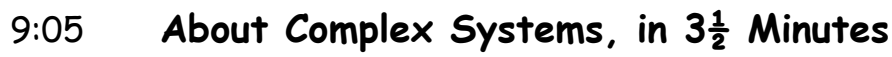

Victor Mossotti, US Geological Survey

9:15 Is the Earth Topography Fractal?

Yaneer Bar-Yam, The New England Complex Systems Institute (NECSI)

9:40 Scaling Properties in the Geosciences

Stephen W. Wheatcraft, Department of Geology, University of Nevada, Reno

10:00 Travel Time for Catchment Flow in a Small Watershed: A New Approach

Based on Fractal Persistence In Stream Tracer Concentrations

Christopher C. Barton \& Gene E. Likens, US Geological Survey

10:30 Coffee

10:50 Self-Dissimilarity: An Empirically Observable Complexity Measure

David H. Wolpert \& William G. Macready, NASA Ames Research Center

11:40 Concentration and Dispersion from Generalized Cantor Sets: Complexity from Simple Operators

Mark E. Gettings, U.S. Geological Survey;

Phillip Hammonds, Northrop-Grumman Information Technology

Bernard P. Zeigler, Arizona Center for Integrative Modeling \&Simulation,

12:00 Explicitly-parallel Implementation of Irregular Problems on SIMD Parallel Computers

Andrea Di Blas, \& Richard Hughey, Dept. of Computer Eng, Univ of California, Santa Cruz

12:20 Lunch 
Day One: Wednesday Afternoon

1:45 Active Walk Model for Positive-Feedback Systems

Lui Lam, Sue Ann Koay, \& My-Phuong Le; Nonlinear Physics Group, Department of Physics, San Jose State University

2:30 Simulation of Rare Spatial-Temporal Events Involving the Interaction of Man and the Environment - Exploration for Mineral Resources

Gary L. Raines; M.L. Zientek; J.D. Causey; and D.E. Boleneus, U.S. Geological Survey

2:50 Modeling Complex Probabilistic Systems for Petroleum and Landslide

Assessments

Robert A. Crovelli, U.S. Geological Survey

3:10 Coffee

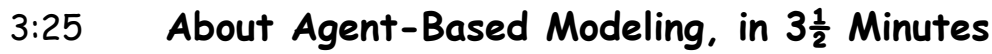

Victor G. Mossotti, U.S. Geological Survey

3:40 Interoperability testing of technology based complex systems

Phil Hammonds, PhD, Northrop-Grumman Information Technology; Bernard P. Zeigler, Arizona Center for Integrative Modeling and Simulation; and Mark E. Gettings, U.S. Geological Survey,

4:00 "DYNASED," A Fully-Coupled Dynamic System Representing Variations in Sea Level, Elevation of Continents, and Rates of Erosion

John W. Harbaugh, Stanford University \& Brian Penn, Autometric, Inc.

4:30 Length and Time Scales of Multicomponent Convection in Porous Media Clay A. Cooper, Division of Hydrologic Sciences, Desert Research Institute, Reno

5:00 End of Wednesday program at Reid Engineering

6:00 Reception: Desert Research Institute, 2215 Raggio Pkwy, Reno

Transportation provided from University Inn 5:45, 6:00, 6:15

7:00 Dinner

8:15 Dinner Keynote Address

STEPHEN WOLFRAM

A NEW KIND OF SCIENCE

Wolfram Research, Inc

Stephen Wolfram will describe ideas and discoveries from his book $A$ New Kind of Science, their implications for various fields of science, and their personal and historical context. An extended auestion and answer period will be included. 
Day Two: Thursday Morning

7:30 Continental Breakfast

8:00 Earthquake Simulation Studies \& Laboratory Tour, UNR

Ian Buckle, Center for Civil Engineering Earthquake Research, University of Nevada, Reno

8:40 Keynote Speaker Lael Parrott, Directrice, Laboratoire de systèmes complexes Géographie, Université de Montréal Using complexity measures to characterize the dynamics of ecosystems: Lessons from modeling

9:40 On The Logical Relationship Between Natural Selection And SelfOrganization

Guy A. Hoelzer, Department of Biology, University of Nevada Reno

10:00 Coffee

10:20 Scale Invariance Modeling Techniques in GeoDAS GIS And Decomposition of Complex Geo-Anomalous Patterns

Qiuming Cheng, York University, Toronto, Ontario, Canada

11:00 Complex System Simulation: Interactions of NOM Molecules, Mineral Surfaces, and Microorganisms in Soils

Gregory Madey, Yingping Huang, Xiaorong Xiang, Eric Chanowich, Patricia MauriceUniversity of Notre Dame, Notre Dame, IN, \& Steve Cabaniss, University of New Mexico, Albuquerque, NM

11:20 Self-organization and complexity in the pelagic marine biological system Dale C. Krause, Marine Science Institute; University of California, Santa Barbara

11:40 Gaps in virtual forests: local-scale ecology studies using a spatiallyexplicit individual-based model.

Ménard, A. ${ }^{1}$, Dubé, P. ${ }^{1}$, Bouchard, $A .^{2}$ and Marceau, D.J. ${ }^{1}$

${ }^{1}$ Geocomputing Laboratory, Université de Montréal, Canada. (menarda@sympatico.ca)

${ }^{2}$ IRBV, Université de Montréal, Canada

12:00 Lunch 
Day Two: Thursday Afternoon

1:00 A Few Classic Examples in Complex Systems and Genetic Algorithms Wolfgang Bein, Dept. of Computer Sciences, University of Nevada, Las Vegas

1:30 Fractal Nature of Hydrocarbon Accumulations; Implications for Resource Assessment and Exploration Strategy

Christopher C. Barton, , U.S. Geological Survey

2:00 Discrete Events - Multi-resolution Modeling And Simulation of SelfOrganizing Criticality In Complex Systems

Bernard P. Zeigler ${ }^{1}$, Mark E. Gettings ${ }^{2}$, Phillip Hammonds ${ }^{3}$

${ }^{1}$ Electrical \& Computer Engineering Dept, University of Arizona, Tucson, AZ,

${ }^{2}$ U.S. Geological Survey, Tucson, Arizona

${ }^{3}$ Northrop-Grumman Information Technology, Ft. Huachuca, Arizona

2:20 Modeling complex behavior in communications networks

Ian Marshall, BT exact Technologies and University College London

2:40 Preliminary Cellular-Automata Forecast of Permit Activity from 1998 to 2010, Idaho and Western Montana

Gary L. Raines; M.L. Zientek; J.D. Causey; and D.E. Boleneus, U.S. Geological Survey

3:00 Coffee

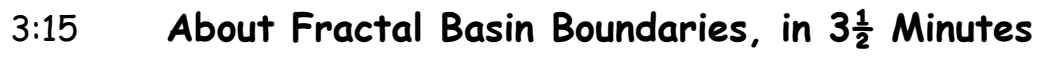

Victor G. Mossotti, U.S. Geological Survey

3:20 The Generalized 20/80 Law Using Probabilistic Fractals Applied to Petroleum Assessment

Robert A. Crovelli, U.S. Geological Survey

3:40 Predicting Permit Activity with Cellular Automata Calibrated with Genetic Algorithms

Sushil J. Louis, Genetic Algorithm Systems Laboratory, University Of Nevada \& Gary L.

Raines, U.S. Geological Survey

4:00 New Directions for Complex Systems R \& D

Zann Gill, Director, MetaVu Network, Los Altos, CA 94024

4:20 Learning Control behavior from complex models

Jack Alpert, Stanford Knowledge Integration Laboratory, Stanford, CA

4:30 End of Thursday program at Reid Engineering 


\section{List of Presentations}

[Day and Session Information in Brackets

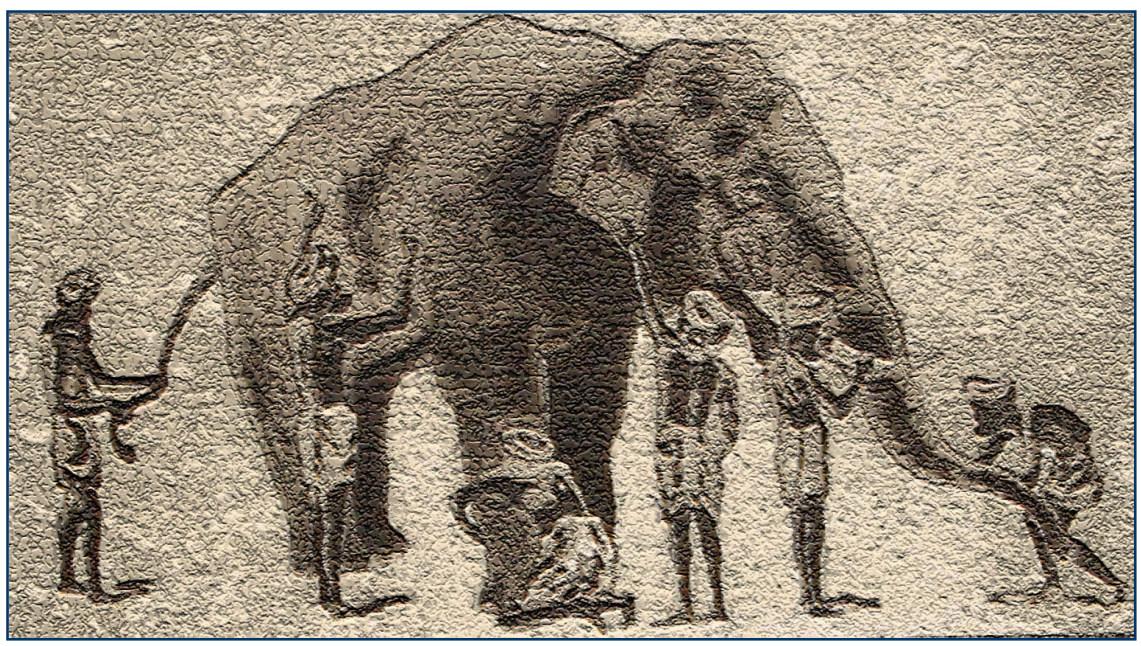

Alpert, [Th, 4:20] Jack Alpert, Learning Control behavior from complex models

Barton, [Th, 1:30] Christopher C. Barton, Fractal nature of hydrocarbon accumulations; implications for resource assessment and exploration strategy

Barton, [W, 10:00 ] Christopher C. Barton and Gene E. Likens

Travel time for catchment flow in a small watershed: a new approach based on fractal persistence in stream tracer concentrations

Bar-Yam, [W, 9:15 ] Yaneer Bar-Yam, Is the Earth topography fractal?

Bein, [Th, 1:00] Wolfgang Bein, A few classic examples in complex systems and genetic algorithms

Buckle, [Th, 8:00] Ian Buckle, Earthquake Simulation Studies \& Laboratory Tour

Cheng, [Th, 10:20 ] Qiuming Cheng, Scale invariance modeling techniques in GeoDAS GIS and decomposition of complex geo-anomalous patterns

Cooper, [W, 4:30 ] Clay A. Cooper, Length and time scales of multi-component convection in porous media

Crovelli, [Th, 3:20 ] Robert A. Crovelli, The generalized 20/80 law using probabilistic fractals applied to petroleum assessment 
Crovelli, [W, 2:50 ] Robert A. Crovelli, Modeling complex probabilistic systems for petroleum and landslide assessments

Di Blas, [W, 12:00] Andrea Di Blas, Richard Hughey, Explicitly-parallel implementation of irregular problems on SIMD parallel computers

Gettings, [W, 11:40 ] Mark E. Gettings, Phillip Hammonds and Bernard P. Zeigler, Concentration and dispersion from generalized Cantor sets: Complexity from simple operators

Gill, [Th, 4:00 ] Zann Gill, New directions for complex systems $R \& D$

Hammonds, [W, 3:40] Phil Hammonds, Bernard P. Zeigler, and Mark E. Gettings, Interoperability testing of technology based complex systems

Harbaugh, [W,4:00 ] John W. Harbaugh and Brian Penn, "DYNASED," A fullycoupled dynamic system representing variations in sea level, elevation of continents, and rates of erosion

Hoelzer, [Th, 9:40 ] Guy A. Hoelzer, On the logical relationship between natural selection and self-organization

Kauffman, [Th, 8:20am] Stuart A. Kauffman (Keynote speaker), Molecular Autonomous Agents: A Possible Definition of Life, And a Possible Technological Revolution

Krause, [Th, 11:20 ] Dale C. Krause, Self-organization and complexity in the pelagic marine biological system

Lam, [W, 1:45] Lui Lam, Sue Ann Koay, and My-Phuong Le, Active walk model for positive-feedback systems

Louis, [Th, 3:40] Sushil J. Louis and Gary Raines, Predicting permit activity with cellular automata calibrated with genetic algorithms

Madey, [Th, 11:00] Gregory Madey, Yingping Huang, Xiaorong Xiang, Eric Chanowich, Patricia Maurice, and Steve Cabaniss, Complex system simulation: Interactions of NOM molecules, mineral surfaces, and microorganisms in soils

Marshall, [Th, 2:20 ] Ian Marshall, Modeling complex behaviour in communications networks 
Ménard, [Th, 11:40] A. Ménard, P. Dubé, A. Bouchard, and D.J. Marceau, Gaps in virtual forests: Local-scale ecology studies using a spatially-explicit individual-based model

Mossotti, [W, 9:05; W, 3:25; Th, 3:15] Victor G. Mossotti, About Complex Systems, in 31/2 minutes; About Agent-Based Modeling, in 31/2 minutes; About Fractal Basin Boundaries, in 31/2 minutes

Parrott, [Th, 9:20] Lael Parrott, Using complexity measures to characterize the dynamics of ecosystems: Lessons from modeling

Raines, [Th, 2:30 ] G.L. Raines, M.L. Zientek, J.D. Causey, and D.E. Boleneus; Preliminary cellular-automata forecast of permit activity from 1998 to 2010, Idaho and Western Montana

Raines, [W, 2:20 ] G.L. Raines, M.L. Zientek, J.D. Causey, and D.E. Boleneus; Simulation of rare spatial-temporal events involving the interaction of man and the environment - exploration for mineral resources

Wheatcraft, [W, 9:40] Stephen Wheatcraft, Scaling issues in the Geosciences

Wolfram [W, 8:15pm] Stephen Wolfram (Dinner Keynote), Wolfram Research, Inc. Stephen Wolfram will describe ideas and discoveries from his book A New Kind of Science, their implications for various fields of science, and their personal and historical context. An extended question and answer period will be included.

Wolpert, [W, 10:50] David H. Wolpert and William G. Macready, Selfdissimilarity: An empirically observable complexity measure

\section{Zeigler [Th, 2:00] Bernard P. Zeigler, Mark E. Gettings, Phillip Hammonds,} Discrete events multi-resolution modeling and simulation of self-organizing criticality in complex systems 


\section{ABSTRACTS \\ (in alphabetical order by first presenter's last name)}

Christopher C. Barton, , U.S. Geological Survey, 600 4th Street, South, St. Petersburg, FL 33704

\section{Abstract: Fractal Nature of Hydrocarbon Accumulations; Implications for Resource Assessment and Exploration Strategy}

Since the early 1960s, the lognormal function had been fit to the size frequency distribution of hydrocarbon accumulations. This presentation will review my work with C.H. Scholz which showed that the fractal power function (not the lognormal) is the correct underlying distribution, fully describing the size-frequency distribution from the largest accumulations to the size below which economic truncation results in an incomplete sampling. The use of the fractal power function results in higher assessments of the size and number of undiscovered accumulations.

The spatial distribution of hydrocarbons in a single stratigraphic unit in the Denver and the Powder River basins is fractal with the same dimension of 1.4. The historic exploration strategy used in these basins approaches a fractal dimension of 2.0, which is equivalent to Poissonian random drilling with no geologic input. 


\section{Christopher C. Barton ${ }^{1}$ and Gene E. Likens ${ }^{2}$}

${ }^{1}$ US Geological Survey, 600 4th Street, South; St. Petersburg, FL 33704

${ }^{2}$ Institute of Ecosystem Studies, Box AB, Millbrook NY 12545

\section{Abstract: Travel Time for Catchment Flow in a Small Watershed: A New Approach Based on Fractal Persistence In Stream Tracer Concentrations}

We calculate the time it takes for precipitation to move through the catchment to the stream in the Watershed-6 at Hubbard Brook, NH. The catchment travel time is a fundamental hydrologic parameter that affects the retention of soluble contaminants in the watershed. Our tracer is $\mathrm{Cl}$, which is conservative. Spectral analysis of the 35 year $\mathrm{Cl}$ concentration time series shows that the fluctuations over three orders of magnitude are fractal with a scaling exponent of 0.5 for precipitation and 0.7 for stream water measured at the weir. Therefore, the $\mathrm{Cl}$ fluctuations in the catchment are necessarily fractal, as are flow paths. A fractal distribution of catchment travel times means that after a contaminant pulse, concentration will initially drop rapidly, but then decline much more gradually and will persist for very long times. This "fat tailed" decay is characteristic of fractal distributions. Flow through the catchment dampens the chloride signal and increases the persistence of the signal, which permits calculation of the average flow time through the catchment. We fit the catchment spectrum with a power form of the Gamma Distribution and calculate the average travel time through the catchment. The average flow time through the catchment is 3.7 months. 


\section{Yaneer Bar-Yam, The New England Complex Systems Institute (NECSI)}

24 Mt. Auburn St., Cambridge, MA 02138, TEL 617-547-4100 FAX 617-661-7711

necsi@necsi.org

\section{Abstract: Is the Earth topography fractal?}

We report the scaling behavior of the Earth roughness over a wider range of length scales than reported by previous researchers. All landscapes (not only mountains) together follow a consistent scaling behavior --- but one that is not described by a single scaling exponent. Instead, it demonstrates a crossover between highly correlated (smooth) behavior at short length scales (with a scaling exponent $\alpha=1$ ) and self-affine behavior at long length scales, above $10 \mathrm{~km}$, with $\alpha=0.4$. An analogous analysis of Venus provides similar results with the long length scale regime above $50 \mathrm{~km}$. The possible implications for geologic dynamics and skiing will be discussed. 


\section{Wolfgang Bein, School of Computer Science,University of Nevada, Las Vegas \\ Abstract: A Few Classic Examples in Complex Systems and Genetic Algorithms}

A system consisting of simple parts which are easily understood can bring about complex behavior when these parts interact. We review a few intriguing examples in areas such as cellular automata, autonomous agents, dynamical systems, competitive games. With complex systems there are interesting connections to genetic algorithms, some of which we will highlight here. 
Qiuming Cheng, Department of Earth and Atmospheric Science, Department of Geography, York University, Toronto, Ontario, Canada, M3J 1 P3

E-mail:qiuming@yorku.ca,Tel: (416)-7362100 ext.22842,Fax: (416)-736 5817

\section{Abstract: Scale Invariance Modeling Techniques in GeoDAS GIS And Decomposition of Complex Geo-Anomalous Patterns}

Spatially superimposed multiple processes such as multiplicative cascade processes often generate complex patterns possessing so-called scale invariance properties that can be described by power-law type of functions within certain scale ranges. The examples related to study of mineral resources may include fractures and faults caused by tectonics and structure activities; distribution of concentration values of trace elements in rocks and soils caused by mineralization; and locations of mineral deposits of different types and sizes etc. While it is useful to characterize these spatial features or spatial patterns by means of fractality/multifractality, it is even more important to be able to differentiate these patterns on the basis of distinct fractal and multifractal properties, for example, to separate anomalous patterns of trace elements caused by mineralization from background values due to normal geological processes. The local mineralization and regional geological processes may cause geochemical patterns with distinct scale invariance properties including variable scaling ranges. Separation of these patterns on the basis of the distinct scaling properties, including scaling ranges, can provide a powerful means for anomaly separation and pattern decomposition. Scaling breaks have been observed from various patterns. This paper introduces a set of newly developed techniques for quantifying anisotropic scale invariance and for selecting scaling breaks to separate complex patterns.

Geographical information systems (GIS) have been commonly used to organize large volumes of spatial data. One of the advantages of using GIS is to integrate different types of spatial data covering the same area but with different coordinate systems, scales and resolutions. It is to visualize changes in spatial data as a function of changes in the scales and resolution. This is often useful for data interpretation, especially for geophysical and geochemical data interpretation. Therefore, GIS naturally needs functionality for quantifying scale invariance properties and for conducting non-linear modeling. Implementing scaling techniques in a GIS environment enables the application of constraints for separation of spatial patterns. For example, 
conditional multifractal measures can be defined on the basis of one type of spatial feature, while additional attributes can be appliedas conditions on the spatial separation of patterns.

A new system GeoDAS (GeoData Analysis System) has been recently developed at York University in collaboration with the Geological Survey of Canada and U.S. Geological Survey for processing, interpreting and integrating diverse exploratory datasets for mineral exploration and environment assessment. In addition to the common functionalities available in commercial GIS, GeoDAS has implemented a suite of new techniques including fractal/multifractal techniques for data visualization, information extraction and data integration. Several datasets will be used to demonstrate the application of GeoDAS for identification of mineralization-associated anomalies from background fields. 
Clay A. Cooper, Div. of Hydrologic Sciences, Desert Research Institute, Reno, NV 89512

\section{Abstract: Length and Time Scales of Multicomponent Convection in Porous Media}

Convection is a type of fluid motion that occurs when a parcel of fluid becomes buoyant to its surroundings causing the parcel to move in the direction of locally decreasing density. In the presence of more than one density-affecting component, however, additional couplings can occur such that organized motion occurs even though the global density gradient is stable. This multicomponent convection phenomenon, well documented as a major contributor to mixing within oceans and stars, has not been adequately investigated within the context of subsurface porous media. The figure below is an example of evolving multicomponent convection in a HeleShaw cell (an apparatus consisting of two parallel glass plates separated by a thin gap, which simulates many characteristics of similar flow in porous media). A layer of sucrose overlies a layer of sodium chloride such that the overall density gradient is stable. Due to a difference in molecular diffusivities of the two solutes (one of the couplings), organized structures develop and transport mass throughout the cell much faster than would occur due solely to molecular diffusion. With time, mixing is complicated by the interaction of adjacent fingers, by "newer" fingers traveling within the conduits of older ones, and by fingers splitting. Little of this behavior is understood quantitatively. In addition to describing the qualitative characteristics of such flows, behavior can be analyzed through integral measures. Results have shown that in highly energetic systems (low buoyancy ratio; a measure of the contribution to overall density of each component) mass flux reaches a steady state, even though the structures continue to evolve. For diffusive-like systems close to the stability boundary, mass flux scales with $t^{-1 / 2}$. This is attractive for an eddytype analysis of diffusion coefficients, although one has not been attempted yet from a theoretical standpoint.

In the context of subsurface flow and transport, the ultimate goal is to understand spatial and temporal asymptotic behavior in heterogeneous porous media in the absence of boundaries. In geological settings, multicomponent convection might be an example of "penetrative convection" where a structured plume (or group of fingers) advances into a fluid of stable stratification. Laboratory studies are limiting in that we have no idea what types of motion occur on length scales larger than $1 \mathrm{~m}$. A simple theory might suggest that the phenomenon should continue to grow as long as the boundary conditions are satisfied. When one considers interactions among the 
fingers, however, little is known of the resulting velocity and component fields, as our understanding of interacting finger dynamics is not well understood. 


\section{Robert A. Crovelli,}

U.S. Geological Survey, Box 25046, MS 939, Denver Federal Center, Denver, Colorado 80225

Phone: (303)236-5770 E-mail: crovelli@usgs.gov

\section{Abstract: Modeling Complex Probabilistic Systems for Petroleum and Landslide Assessments}

Two complex probabilistic systems are discussed for (a) petroleum resource assessment and (b) landslide hazard assessment. The two systems have the same basic mathematical structure even though the subject matter and operational details of the two disciplines are very different. The common basic relationship involves the sum of a random number of random variables.

The Analytic Cell-based Continuous Energy Spreadsheet System (ACCESS) was designed from analytic probabilistic methodology to calculate estimates of oil and gas resources in continuous (unconventional) petroleum accumulations. The analytic probabilistic methodology was developed by deriving the necessary mathematical equations based upon conditional probability theory and laws of expectation and variance. Two important random variables in the system are (1) number of potential untested cells and (2) total recovery per cell. The analytic probabilistic methodology was used to construct a computer spreadsheet program. The ACCESS spreadsheet is applied to estimate the potential additions to reserves of oil and gas in a continuous assessment unit, within a total petroleum system, for the present USGS National Oil and Gas Assessment.

The Probabilistic Landslide Assessment Cost Estimation System (PLACES) was designed from analytic probabilistic methodology to calculate estimates of number and economic loss (cost) of landslides during a specified time. Two important random variables in the system are (1) number of landslide clusters and (2) cost per landslide cluster. The analytic probabilistic methodology was used to construct a computer spreadsheet program. The PLACES spreadsheet is applied to estimate the damage, as economic loss, from future landslides caused by rainstorms in the San Francisco Bay region, California. 


\section{Robert A. Crovelli,}

U.S. Geological Survey, Box 25046, MS 939, Denver Federal Center, Denver, Colorado 80225

Phone: (303)236-5770 E-mail: crovelli@usgs.gov

\section{Abstract: The Generalized 20/80 Law Using Probabilistic Fractals Applied to Petroleum Assessment}

The 20/80 law is a heuristic law that has evolved over the years into the following rule of thumb for many populations: 20 percent of the population accounts for 80 percent of the total value. The general $p 100 / q 100$ law in probabilistic form for a random variable is developed with $q$ as a function of $p$, where $p$ is the population proportion and $q$ is the proportion of total value.

Using the Pareto distribution, the $p 100 / q 100$ law in probabilistic form is derived with the parameter $q$ being a fractal, where $q$ possesses the scale invariance property. The Pareto form of the $p 100 / q 100$ law is applied to petroleum field-size data to obtain $p$ and $q$ such that $p 100 \%$ of the oil fields greater than any specified scale or size in a geologic play account for $q 100 \%$ of the total oil of the fields. The theoretical percentages of total resources of oil using the Pareto $q$ are extremely close to the empirical percentages from the data using the statistic $q$. Also, the empirical scale invariance property of the statistic $q$ for the petroleum field-size data is in excellent agreement with the theoretical scale invariance property of the Pareto $q$.

Using the lognormal distribution, the $p 100 / q 100$ law in probabilistic form is derived with the parameter $q$ being a fractal, where $q$ unexpectedly possesses the scale invariance property. The lognormal form of the $p 100 / q 100$ law is applied to two populations of petroleum estimates. In the USGS 1995 National Assessment of oil and gas resources the onshore areas and adjoining State waters of the Nation were assessed. As part of the 1995 National Assessment, 274 conventional oil plays and 239 conventional nonassociated-gas plays were assessed. The two datasets of estimates studied herein are the following: (1) the mean, undiscovered, technically recoverable oil resources estimated for each of the 274 conventional oil plays, and (2) the mean, undiscovered, technically recoverable gas resources estimated for each of the 239 conventional nonassociated-gas plays. It was found that the two populations of petroleum estimates are both distributed approximately as lognormal distributions. In both cases the theoretical percentages of total resources 
using the lognormal form of the $p 100 / q 100$ law are extremely close to the empirical percentages from the oil and nonassociated-gas data. 


\section{Andrea Di Blas, Richard Hughey}

Dept. of Computer Engineering, University of California, Santa Cruz

\section{Abstract: Explicitly-parallel Implementation of Irregular Problems on SIMD Parallel Computers}

The need for computing performance, not only in scientific computing, but in all kinds of fields, is generating a renewed interest in parallel architectures, and especially in Single InstructionMultiple Data (SIMD) computers. SIMD computers potentially offer the largest computing power at the lowest cost, but represent a difficult obstacle in terms of programming effort. Problems and algorithms with a regular structure and synchronous execution naturally map onto SIMD architectures. Traditionally, however, it has been more common to develop algorithms for irregular, general problems on the more flexible Multiple Instructions-Multiple Data (MIMD) architecture. Nevertheless, considerable effort has been devoted to explore methods to map asynchronous, general problems onto the powerful SIMD architecture. The common opinion is that SIMD computers actually can efficiently solve irregular problems, but to the best of our knowledge, there is not a clear programming methodology that can be applied in general.

Here we describe a methodology to implement asynchronous, irregular applications on distributed-memory SIMD parallel computers. Our methodology, the SIMD Phase Programming Model (SPPM), is a new approach to bridging the gap between the simplicity of implicit parallel programming and the efficiency of explicitly parallel programs. In this approach, the programmer explicitly defines the partitioning of the problem and the degree of parallelism, but unlike other approaches this methodology does not need any high-level supervision for dynamic task allocation and load balancing. Instead, the flow control is split between the controller and the individual processing elements, while fully respecting the SIMD paradigm. Low-cost optimization techniques are used that dramatically improve performance by reducing parallelism overhead, by far the major cause of performance loss intrinsic to our model.

Case studies exemplifying the methodology are presented, with implementations on two different SIMD architectures (UCSC Kestrel and MasPar MP-2). Our experimental results show good performance with respect to serial CPU run time and in terms of high parallel speedup and efficiency. 
Finally, our current investigation shows that optimization -so crucial to performance- can be implemented automatically and can be run-time adaptive, rather than the job of the programmer. This would offer a self-optimizing coding methodology for a broad range of applications on SIMD architectures and could become an efficient compiler technique for implicitly parallel languages. 


\section{Mark E. Gettings, U.S. Geological Survey, Tucson, Arizona \\ Phillip Hammonds, Northrop-Grumman Information Technology, Ft. Huachuca, Arizona}

Bernard P. Zeigler, Arizona Center for Integrative Modeling \&Simulation, www.acims.arizona.edu

\section{Abstract: Concentration and Dispersion from Generalized Cantor Sets: Complexity from Simple Operators}

The Cantor set can be viewed as a conservative system in which the contents are distributed into ever-decreasing size line segments by putting half the contents of a line segment into the first and last thirds of the segment and zero into the middle third. In the limit the set becomes one of zero measure, with an infinite number of members of infinitesimal size, each of infinite density. If the generation of the set is stopped at a finite number of steps (finite scale), the process models concentration of the contents. The process can be generalized into unequal length segments and unequal (and nonzero) proportions allocated to each segment. This defines a general multiplicative process in which the final content of each segment is a product of the proportions from all the steps or generations of line division in the final distribution. If the order of applying the division and proportioning is randomized, distributions statistically identical to real distributions observed in earth science are obtained. Two examples of application of the concentration model are the distribution of tonnage of ore as a function of deposit grade, and the distribution of magnetic susceptibility in hydrothermally altered rocks in porphyry systems. . In the latter model, the line division process determines the distribution of fractures. The susceptibility of interfracture blocks is determined by oxidation of the wallrock, a process controlled by the diffusion equation. Multiple processes can be modeled by serial application of line division processes. The concentration of selenium in the San Francisco Bay ecosystem can be modeled by such a series of superposed line division models. The resulting distributions are complex, and resist inversion to yield the concentration process parameters.

The logical opposite of the concentration process is dispersion. The dispersion processes considered here are not the inverse of concentration model, as they are additive rather than multiplicative processes. The dispersion process is viewed as an operator applied at particular points in space chosen by rules (e.g., randomly. In this form, the operator models erosion or mass movement, due for example to rain and wind. The application of the "wind" and "rain" operators to a model landscape result in complex distributions of the sedimentary detritus which closely 
resemble real distributions of sedimentary fill within a basin when particle size (cobbles, sand, and fines) are accounted for. When combined with a fracturing model (a concentration processes discussed above) to define the distribution of canyons in the source ranges, a model for the distribution of coarse-, medium- and fine-grained detritus in a basin can be achieved that is statistically similar to observed distributions of basin fill. 
Zann Gill, Director, MetaVu Network, PO Box 4001, Los Altos, CA 94024

650-917-1612 zanngill@metavu.net

\section{Abstract: New Directions for Complex Systems R \& D}

I will provide a brief introduction for a panel session and open discussion on opportunities for collaborative projects spinning from the workshop and on the design of a collaborative web environment to facilitate post-workshop follow-up.

The intent of the panel session is to explore how the workshop could become a springboard for the development of collaborative $\mathrm{R}$ and $\mathrm{D}$ projects addressing the research priorities of USGS, NASA, DRI, participating universities, etc. I will solicit input from the group into the design of a system to support workshop follow-up.

One possibility is to develop a simple "webtank" (think tank on the web) environment to post a first draft summary of outcomes and format into which attendees can contribute their ideas. The intent would be to develop one or more outstanding collaborative projects that can secure funding to proceed. 


\section{Phil Hammonds, Northrop-Grumman Information Technology, Ft. Huachuca, AZ Bernard P. Zeigler, Arizona Center for Integrative Modeling and Simulation; www.acims.arizona.edu}

Mark E. Gettings, U.S. Geological Survey, Tucson, AZ.

\section{Abstract: Interoperability testing of technology based complex systems}

The analysis of complex systems including physical, biological, social and technological systems is subject to errors induced by observer perspective, choice of scale and misunderstanding of the context of system function. As we move from elements to components to systems and then to systems of systems and ultimately to super-systems, the propagation and compounding of error may not be recognized prior to or during analysis.

Interoperability testing of technology based complex systems, such as military Command, Control, Communications, Computers, Intelligence, Surveillance and Reconnaissance (C4ISR), systems of systems requires careful consideration of scale, perspective and context of function. Interoperability of such systems is essential for effective military operations, but the service specific environments in which these systems were designed often limit interoperability even though these systems were built to standards accepted by all the services. Building to standards does not guarantee interoperability.

Military systems of systems interoperability testing has progressed from the testing of conformance to standards, i.e., protocols, to the testing of procedural and content standards, i.e., timing and message formats, to the present state of systems of systems interoperability testing. We are presently testing systems that were not originally intended to work together, but must now interoperate in real-world high-stakes conditions. We have reached a level of complexity where we must carefully examine our assumptions of how interoperability tests are designed and conducted before we can determine whether we are actually testing a characteristic we describe as interoperability. Testing methodology becomes a critical issue and further suggests that we pay careful attention to the design of models and simulations that will inform us of potential sources of error before we begin actual testing. 
Comparisons of the behavior of these technologically complex systems of systems to physical, biological and social systems will be examined in order to improve understanding of observationally induced error in complex systems analysis. 


\section{John W. Harbaugh ${ }^{1}$, Brian Penn ${ }^{2}$}

1Professor Emeritus, Geological and Environmental Sciences Dept., Stanford University harbaugh@pangea.stanford.edu

2Autometric, Inc. A Business Unit of the Boeing Company, Colorado Springs, CO. 80910, brian.penn@csautometric.com

\section{Abstract: "DYNASED," A Fully-Coupled Dynamic System Representing Variations in Sea Level, Elevation of Continents, and Rates of Erosion}

Most dynamic systems exhibit cyclic and chaotic behavior, but few attempts have been made to represent the Earth's crust as a dynamic system in which the components are linked together so each component depends on all the other components. By drastically simplifying things, the components and their interdependencies can be represented by equations that can be solved numerically. The behavior of the system can be examined by running it for several million cycles in each of a series of individual experiments.

"DYNASED" is thus a "fully-coupled" system where three equations link three "state" variables together. The state variables are (1) the general elevation of the continents, (2) sea level, and (3) the rate at which sediment eroded from the continents is transferred to the sea. Each state variable represents the state of the system at any instant. While DYNASED is hugely simplified with only three state variables, it usually responds with astounding complexity and unpredictability when the control parameters (which consist of coefficients, exponents, and lags) are adjusted at the outset of an experiment.

Control parameters are not variables in the usual algebraic sense, but in practice they can be varied so that the behavioral properties of the system can be explored. With a specific setting of control parameters, DYNASED may respond for many thousands of iterations with a high degree of cyclicity, with lesser cycles nested within successively greater cycles. Suddenly, however, it may drift from more or less "predictable" cyclicity to extreme chaos, and then return later to cyclicity, and even to quasi-stability. Experiments spanning many millions of iterations are feasible.

While artificially simple, DYNASED suggests that the Earth's crust and its lesser component systems may behave autocyclically, so that external "forcing functions" are unnecessary even 
though drastic changes in behavior occur. One general conclusion is that creation of fully-coupled mathematical models of dynamic systems is likely to change our outlook in geology. The responses of the models are likely to be counter-intuitive because cause-and-effect relationships are blurred.

Although DYNASED is essentially one dimensional (the one dimension represents variations in sea level and variations in the general elevation of the continents), its "geological" responses may be viewed as two-dimensional stratigraphic cross sections in which dispersion of clastic sediment is represented by the diffusion equation, and in which dispersion rates are sensitive to the rate at which sediment is supplied to the sea at continental margins, which in turn are sensitive to variations in the difference between sea level and the general elevation of continents. The idea is to see how features of actual stratigraphic sequences formed at continental margins might correspond to those produced in simulations by DYNASED. The implied assumption is that DYNASED's drastically simplified formulation preserves a degree of realism; this assumption is yet to be tested. 


\section{Guy A. Hoelzer, Department of Biology, University of Nevada Reno, hoelzer@unr.edu}

\section{Abstract: On The Logical Relationship Between Natural Selection And Self-Organization}

Biological evolution is often cited as an example of self-organization in a dynamical complex system, which is consistent with the notion that self-organization continually optimizes structures in response to unpredictable perturbations. Some authors have even suggested that this model of adaptive evolution should be considered as an alternative to Darwin's model of adaptation through natural selection. There is, however, no reason to think that these are mutually exclusive processes. In this talk, life will be described as a self-organized engine that reduces an energy gradient created by the earths shadow; the constraints of birth/reproduction/death increase the "coarseness of the grains" of the system, permit better optimization of energy flow, and provide the foundation for natural selection among phenotypic variants to occur. This self-organizing engine can then take advantage of natural selection as a means of further optimizing energy flow. This view is also consistent with the claim that NS takes advantage of the self-organizing tendencies of physical systems at smaller scales. For example, the self-organizing aspects of organismal development permit phenotypes to be economically encoded in genomes.

Conversely, scales of functional organization above that of individuals, such as ecosystems, can take advantage of natural selection as a means to self-organization. The potential for obtaining novel insights by considering the physical basis of natural selection is illustrated by data recently compiled by Moses and Brown, who show strong and predictable relationships between energy processing and fitness (fecundity) among and within human cultures, as well as among biological species. In summary, NS is described as an emergent process founded on physical thermodynamics. This view places Darwin's theory into a more general, physical framework, and points the way to understanding biological evolution and adaptation as manifestations of the same physical laws that govern non-living systems. 
Dale C. Krause, Marine Science Institute; University of California, Santa Barbara, CA 93106. krause@geol.ucsb.edu.tel: 805-893-7237

\section{Abstract: Self-organization and complexity in the pelagic marine biological system}

The pelagic marine biological system (MBS) is driven by the physical and chemical processes of the ocean and its interfaces, and the organisms have evolved to take advantage of those processes. The complex system is characterized by power-law and other nonlinear interactions between its components and processes in space and time. Thus the MBS is considered to be self-organized. A key driving factor of the system is that of the power-law ocean turbulent processes, ranging from large scale $(100 \mathrm{~s}$ of $\mathrm{km})$ to small scale $(\mathrm{mm})$, having a fractal dimension of $\mathrm{D}=1.67$. A laminar flow regime operates at smaller scales. Significant biological consequences are shown at all scales. The self-organized complexity is illustrated by three examples involving the food web: (1) The patchiness of western North Atlantic phytoplankton has a power-law fractal dimension similar to that of turbulence, due to passive distribution by the turbulence, as measured over a season at an oceanographic buoy near Bermuda. (2) Northwestern Pacific herbivore copepods graze on phytoplankton and have a patchiness with a higher fractal dimension near $\mathrm{D}=1.8$ (Tsuda, 1995), inferred to be the combined result of turbulence and swimming by random walk. (3) Eddies can be considered to be semi-contained ecosystems, in essence self-organized; Antarctic krill swarms are inferred to be partially organized by ocean eddies ( $\mathrm{D}=1.67)$, upon which is superimposed krill feeding behavior (feeding on phytoplankton) involving swarming, with the swarm sizes having a fractal dimension near $\mathrm{D}=1.9$. Although present numerical and box modeling approaches give significant insights into the functioning of the MBS, these approaches are hampered by their specialized conceptual frameworks, the need to use linear approximations of the nonlinear processes and relationships, and the limitations in available computer power. In addition, major knowledge gaps exist, such as that concerning the gelatinous organism component of the food web, which is inadequately understood and modeled. The present stepwise approach to dealing with the inherent complexity of the MBS needs to be augmented by an overall research strategy. For example, the models need to be developed to reflect adequately the self-organizing nature of the system, and the size-scaling and energy/mass budgeting in the food web. Nevertheless important progress is being made, and the paradigm shift is evolving rapidly. 


\section{Lui Lam, Sue Ann Koay, and My-Phuong Le}

Nonlinear Physics Group, Department of Physics, San Jose State University, San Jose CA 95192-0106

\section{Abstract: Active Walk Model for Positive-Feedback Systems}

Active walk (AW) is a paradigm for pattern formation and self-organization in complex systems, proposed by us in 1992 [Lam 1997; Lam 1998]. In an AW, the walker changes the landscape as it walks and is influenced by the changed landscape in choosing its next step. Active walk models have been applied successfully to various biological, physical, geological and financial systems in both natural and social sciences, including positive-feedback systems. Examples of the latter consist of convection in the atmosphere or over sand surface, evolution of life, ecosystems, disease outbreak, age-structured population, and economics. Here, a solvable AW model, in which a single active walker is allowed to jump between two sites, is presented. The probability that a site is picked by the walker depends on the height of the potential (the fitness) at this site. When the ratio of the probabilities of picking the two sites depends only on the height difference, the model is found to be equivalent to a one-dimensional position-dependent probabilistic walk. The latter is then solved analytically, with results in agreement with our computer simulations. The two-site AW model is used to explain the competition between two products in the market, in which the active walker plays the role of the consumer. The inferior product may or may not win out, depending on contingency and the initial advantage or disadvantage of the product in the market.

\section{References}

Lam, Lui (ed), Introduction to Nonlinear Physics, Springer, New York, 1997.

Lam, Lui, Nonlinear Physics for Beginners, World Scientific, River Edge, NJ, 1998.

Lui Lam and My-Phuong Le, Nonlinear Physics Group, Department of Physics, San Jose State University, San Jose CA 95192-0106.

(luilam@email.sjsu.edu; MyPhuong_Le@yahoo.com.)

Sue Ann Koay, Department of Physics, University of California, Santa Barbara, CA 93106 (present address). 


\section{Gregory Madey, Yingping Huang, Xiaorong Xiang, Eric Chanowich, Patricia Maurice-University of Notre Dame, Notre Dame, IN Steve Cabaniss, University of New Mexico, Albuquerque, NM}

\section{Abstract: Complex System Simulation: Interactions of NOM Molecules, Mineral Surfaces, and Microorganisms in Soils}

Natural organic matter (NOM) is a heterogeneous mixture of organic molecules that is ubiquitous in terrestrial and aquatic ecosystems. NOM plays a vital role in many ecological and biogeochemical processes. NOM, microorganisms, and mineral surfaces can interact to form a complex system with emergent properties, i.e., system properties not present in the parts, but present in the whole system. To better understand these complex systems and the mechanisms leading to emergent properties, we are developing a Web-based stochastic simulation of NOM, mineral surfaces, and microbial interactions, starting with a simple model soil system. Individual molecules, their transport through soil pore waters, adsorption to surfaces, various reactions, chemical and physical attributes and behaviors are simulated using the Java programming language and the SWARM agent-based modeling library (open source software developed at the Santa Fe Institute), Molecules and microorganisms are modeled as heterogeneous agents with individual properties and behaviors. Molecular properties modeled include elemental composition and major functional groups. Currently 10 firstorder and second-order reaction types, sorption to and from mineral surfaces, diffusion, and aqueous transport are the types of behaviors modeled. The simulation is configured, started, and monitored from standard Web-browser pages over the Internet. Configuration of the system includes selecting from standard predefined NOM molecule types and using a "molecular editor" to define new molecular configurations. Each scientist using the simulation has access to a workspace in which every simulation setup, stored molecular configurations, simulation events, and results are stored in a database, and analyzed using standard query commands. Queries can be applied against one or more simulations and reported both in alphanumerical and graphical output. We are designing an online collaboratory built around a data warehouse in which collaborating researchers will merge their simulation data and use data-mining techniques for knowledge discovery, including the discovery of self-organizing emergent phenomena. We use Java Server Pages, Servlets, and Java Database Connectivity protocols to build the Web interface. Our ultimate goal is to make the simulation widely available over the Internet, for interactive use. To provide for scalable reliable service, a loosely coupled infrastructure of application servers and database servers using failover, checkpointing, and automatic restart is implemented. 


\section{Ian Marshall, $B$ T exact Technologies and University College London}

\section{Abstract: Modeling complex behaviour in communications networks}

It is often said that one of the most complex machines built by mankind is the global telecommunications network. Certainly if one's definition of complexity accounts for number of components (billions), number of nodes ( 100k) or degree of connectivity (2-2000) the communications network is extremely complex. It should therefore not be surprising that it exhibits a variety of "symptoms" of complexity including power law connectivity (Barabasi), small world traverses, Fractal Traffic (Bestavros), emergent behaviours (AT\&T Brownout), indeterminate fault conditions, etc.

Historically, modelling of communications networks has been based on assumptions of predictable behaviour, including Poisson traffic that aggregates to a smooth continuum in the core network, linear scaling rules, and independent faults. Clearly these assumptions are over simplifications, but whilst the network was mainly offering telephony, and was only growing slowly, they gave results that were useful. A large body of tools and expertise therefore built up using these simple assumptions. However, the emergence of the Internet has pushed network operators into an era of rapid (exponential) growth of capacity (that exposes the limitations of simple scaling rules), orders of magnitude more burstiness (that can no longer be treated as representing exceptional transients), and much more complex interdependence relationships between services (more than just telephony). Network operators are therefore starting to explore novel approaches to system modelling based on the ideas developed in the complex systems community in the last 20 years.

For example it is now routine to include a fractal traffic generator in many models to assess sensitivity to burstiness, and multi-agent or cellular automata models are common in advanced telecommunications research. There are also a few groups (e.g. Dorigo, Marshall) exploring models of self-organizing networks, protocols and management systems. Acceptance of the results by operational engineers has proved to be a major barrier however. Reasons include:

1. The techniques are largely statistical in nature and do not offer rigorous proofs of stability or absolute bounds on behaviour,

2. The mode of thought required is novel and many engineers feel threatened by the implied need to retrain, or simply prefer to stay with the tools they know,

3. Engineer's and manager's intuitions about complex network systems are often incorrect, and at variance with the model results. 
In contrast, we have found that intuitions regarding natural complex systems are often highly sophisticated. We are therefore making extensive use of natural analogies in our current research. However care must be taken to avoid confusion - many biological analogies have associations with disease or pests, and arouse unintended emotional reactions that distract the audience and reduce the chances of acceptance. A more neutral source of analogy is in geophysical systems, such as rivers, metamorphic reaction fronts, oceanic currents etc. As a result our current research borrows heavily from geophysical analogies. For example we are developing energy efficient routing algorithms for mobile ad hoc sensor networks based on tidal currents, algorithms for autonomous placement of network services based on hydrothermal alteration at diffusion reaction fronts, and algorithms for prioritizing traffic based on fractional crystallization. We believe that our approach will be of interest to other scientists working in these and related areas, and that there may be mutual benefits to be obtained from future collaboration. 


\section{A. Ménard ${ }^{1}$, P. Dubé ${ }^{1}$, A. Bouchard ${ }^{2}$ and D.J. Marceau ${ }^{1}$ \\ ${ }^{1}$ Geocomputing Laboratory, Université de Montréal, Canada. (menarda@sympatico.ca) \\ ${ }^{2}$ IRBV , Université de Montréal, Canada}

\section{Abstract: Gaps in virtual forests: local-scale ecology studies using a spatially-explicit individual-based model.}

One of the key factors of ecosystem dynamics and richness that complex systems theory has highlighted in the last few decades is local interactions between the basic elements of systems. These interactions, expressed in an explicit spatio-temporal manner, allows for emergent properties and self-organization at larger scales. This situation has favored the creation and popularization of a multitude of individual-based models in a variety of scientific domains, including forest ecology. With increasing computing capabilities, the models have become more and more detailed. This is the case of the SORTIE model, a spatially-explicit individual-based forest succession model of the North-Eastern deciduous forest of North America.

The present study has two objectives. First, the potential of SORTIE for local-scale studies is assessed. More specifically, our observations focus on the sensitivity to initial conditions (two initial spatial configurations) of the local scale spatial patterns and on the species sensitivity to introduced disturbances (three gap sizes at two different time steps of introduction). Second, the impact extent of gaps at their periphery is characterized. The sapling growth response to gaps (release episodes) of different sizes (500 to $2000 \mathrm{~m} 2$ ) is used as an indicator of the impact extent.

Our results reveal that the local spatial pattern of each species is not sensitive to different initial spatial arrangement after 250 years. Local scale studies can therefore be executed after this initial period. The results also demonstrate that the species densities are not significantly altered by the introduction of gaps of different sizes and at different time steps. The disturbed local dynamics expressed by the simulated forests are consequently the result of a stable system and not one transiting towards a new dynamics. As for the second objective, the release episodes were analyzed in terms of spatial orientation and distance from gap edges. Results indicate that gap effect extends significantly into the adjacent forest. Release episodes mostly appear in the first 20 $\mathrm{m}$ from gap edges and their orientations are concentrated in the northern hemisphere. Based on different degrees of release occurrence, new gap areas are defined and compared to areas from gap existing definitions. The differences are substantial and may have important implications for forest managers and ecologists. 


\section{Victor G. Mossotti}

US Geological Survey, 345 Middlefield Rd, MS-901, Menlo park, Ca 94025

\section{Abstract: About Complex Systems}

It is often repeated in the popular press that the theory of complexity is the most exciting scientific innovation since quantum mechanics, a sentiment of which I fully agree. The only hitch is that the theory of complexity does not exist, except possibly at the most primitive level. After all, what is a complex system? It would seem that to know of what you speak, that is, to have a theory of something, you would need to have a definition of that thing. Many definitions of complex systems have been advanced over the past two decades. At the subjective end of the definitional spectrum, one is told that "Complex systems are like alligators, you know one when you see one," while on the objective side, one is instructed "complex systems are scale-free in information space." What is it about the properties of a complex system that make it's definition so elusive? And why is it that complex systems are frequently misclassified as a simple system when it comes to modeling?

I will argue in this brief talk that, for the purposes of modeling, it is not sufficient to simply consider the spatial-temporal properties of a system when seeking to classify the system. One must also consider the properties of the system structure (components, links, energy flows, constraints, possible states, etc) and the processes driving the system dynamics (scale, iteration, feedback, linearity-or lack thereof, etc). I will highlight and briefly discuss the main features shared by many complex systems. Although the list may not be complete, the elements of the list are easy to grasp and are essentially without contention.

When it comes to the definition of life, "You know it when you see it" has served the life sciences fairly well for the past two centuries. Maybe the alligator-based definition of a complex system isn't such a bad start after all. 


\section{Victor G. Mossotti}

US Geological Survey, 345 Middlefield Rd, MS-901, Menlo park, Ca 94025

\section{Abstract: About Agent-Based Modeling}

Agent-based modeling (ABM) is a programmable environment for the investigation of complex systems in the natural and social sciences. The system is well suited for modeling the temporal and spatial dynamics of systems comprised of interacting elements. Researchers symbolically instruct communities of artificial, autonomous, interacting "agents" on how to interact with other agents and with an artificial environment. Rather than representing the interactions with differential equations, the researcher typically establishes sets of rules, which govern micro-level interactions. The system dynamics, including macro-level spatial and temporal patterns, is viewed as an emergent property of the community of agents.

In keeping with the pedagogical focus of this talk, I will contrast agent-based modeling with classical modeling methods for earth science problems, and I will provide perspective on the challenge of modeling geological phenomena, either by classical means or with agent-based modeling techniques. To show how agent-based modeling works, I will demonstrate five agentbased simulations of non-linear systems. The first three simulations, traffic jam, predation, and chemical separation, involve inflows and outflows of agents. In the final two simulations, both melt crystallizations models, materials are conserved; all simulations are from Wilensky: http://ccl.northwestern.edu/netlogo/models/.

The ambition of geological research is the understanding of earth processes. In mineral exploration and geologic hazard studies (earthquakes, landslides), an important concern is the application of such understanding for the prediction of mineral deposits or the occurrence of a specific event. While conceptual modeling is a critically important tool in such research, the complexity of the processes precludes the use of classical mathematical methods as a means of prediction. Moreover, a fundamental finding in complex systems science is that prediction of a given phenomenon is often impossible in non-linear iterative systems, even if classical deterministic equations can be written to represent the system dynamics. It follows that if you cannot predict an outcome from a given set of initial conditions, you probably cannot invert a specific outcome to replicate initial conditions. 
So where does agent-based modeling fit in? Agent-based modeling depends on identifying simple rules that govern the interactions between system components. Because of this inherent simplicity, and because massively parallel computations can be done on desktop computers, many problems that would otherwise be intractable by classical methods are now amenable to the agent-based approach. The advantages of agent-based simulations over classical methods include:

- Scale considerations are explicit in the model.

- Relationships are intuitive and simple.

- Computations are numerically tractable.

- Results are graphical in space and time.

- Unanticipated outcomes - e.g., emergent complexity, emergent simplicity, and phase transitions - are natural to the simulation.

- Results are amenable to analysis by statistical physics tools.

Although patterns produced by agent-based simulations cannot be inverted to replicate specific spatial geological patterns (e.g., magnetic susceptibility, gravity, fracture, or geochemical), the simulations can provide insight into the range of dynamic diversity that may be possible for a given class of geologic systems. 


\section{Victor G. Mossotti}

US Geological Survey, 345 Middlefield Rd, MS-901, Menlo park, Ca 94025

\section{Abstract: About Fractal Basin Boundaries}

An ecological principle introduced in the early 1970s states that complex ecosystems are generally less fragile than relatively simple ecosystems. The fragility of the Arctic tundra is often cited as an example of this principle. In stark contrast to this well demonstrated principle, chaos theory, a component of complex systems science, predicts that deterministic systems with nonlinear constraints are prone to exhibit hypersensitivity to perturbations rather than stability. In this brief talk I will discuss a universal class of system dynamics that can reconcile these seemingly contradictory observations.

In order to introduce the concepts of phase space, attractor basins, and fractal basin boundaries, I will make use of a computational simulation based on Newton's map of the nth root of unity, i.e., the roots of $\mathrm{F}(z)=z^{\mathrm{N}}-1$. But before introducing the results of this computation, I will demonstrate a swinging magnet pendulum as a physical analog of the $z^{\mathrm{N}}-1=0$ model to make the computation less abstract. From computer and physical simulations, it will be apparent that attractor basin dynamics are insensitive to perturbation, whereas the dynamic trajectory of the system is hypersensitive to conditions near fractal basin boundaries.

The significant finding is that systems driven by a multiplicity of processes with spatially overlapping fields-of-influence (potential fields) can spawn a variety of Euclidean and scale-free patterns. Although the primary intention of this talk is pedagogical, I will briefly introduce the application of the Newton $/ z^{\mathrm{N}}$ model for understanding a wide class of fractal patterns in geochemistry, Quaternary geology, and ecology. 
Lael Parrott, Directrice, Laboratoire de systèmes complexes Géographie, Université de Montréal

\section{Abstract: Using complexity measures to characterize the dynamics of ecosystems: Lessons from modeling}

As society begins to pose more and more questions regarding the impact of human activities on the Earth's life support systems, and more specifically on the structure and functioning of the ecosystems in which we live, there is an increasingly urgent need to be able to effectively measure and characterize the dynamics of an ecosystem.

With this as our objective, we investigate the effectiveness of different measures of complexity at characterizing the global level dynamics of modeled ecosystems. All simulations are performed with the model WIST (Weather driven, Individual based, Spatially explicit, Terrestrial ecosystem model). WIST is a deterministic, rule-based model that has been shown to exhibit persistent spatiotemporal self-organization in simulated ecosystems. We compare several different simulations based on multi-species configurations subjected to increasing degrees of disturbance. Based on analysis of biomass history in the modeled ecosystems, we demonstrate a relationship between ecosystem complexity and disturbance level. In addition, we propose that the method of recurrence analysis may be a good method of detecting a changing dynamical regime before such change is detectable in a time series. Such analytical methods may bring us one step closer to unraveling the complexity of natural ecosystems. 


\section{G.L. Raines ${ }^{1}$, M.L. Zientek², J.D. Causey ${ }^{2}$, and D.E. Boleneus ${ }^{2}$ U.S. Geological Survey ${ }^{1}$ MS 176 clo Mackay School of Mines UNR, Reno, NV89557 graines@usgs.gov \\ ${ }^{2} 904$ W. Riverside Ave., Room 202, Spokane, WA 99201}

\section{Abstract: Preliminary Cellular-Automata Forecast of Permit Activity from 1998 to 2010, Idaho and Western Montana}

For public land management in Idaho and western Montana, the U.S. Forest Service (USFS) has requested that the USGS predict where mineral-related activity will occur in the next decade. Cellular automata (CA) provide an approach to simulation of this human activity. Cellular automata are defined by an array of cells, which evolve by a simple transition rule, the automaton. Based on exploration trends, we assume that future exploration will focus in areas of past exploration. Spatial-temporal information about mineral-related activity, i.e. permits issued by USFS and Bureau of Land Management (BLM) in the last decade, and spatial information about undiscovered resources, provide a basis to calibrate a CA. The CA implemented is a modified annealed voting rule that simulates mineral-related activity with spatial and temporal resolution of one $\mathrm{mi}^{2}$ and 1 year based on activity from 1989 to 1998 . For this CA, the state of the economy and exploration technology is assumed constant for the next decade. The calibrated CA reproduces the 1989-1998-permit activity with an agreement of $94 \%$, which increases to $98 \%$ for within one year. Analysis of the confusion matrix and Kappa correlation statistics indicates that the CA underestimates high activity and overestimates low activity. Spatially, the major differences between the actual and calculated activity are that the calculated activity occurs in a slightly larger number of small patches and is slightly more uneven than the actual activity. Using the calibrated CA in a Monte Carlo simulation projecting from 1998 to 2010, an estimate of the probability of mineral activity shows high levels of activity in Boise, Caribou, Elmore, Lincoln, and western Valley counties in Idaho and Beaverhead, Madison, and Stillwater counties in Montana, and generally low activity elsewhere. 


\section{G.L. Raines ${ }^{1}$, M.L. Zientek², J.D. Causey ${ }^{2}$, and D.E. Boleneus ${ }^{2}$ U.S. Geological Survey \\ ${ }^{1}$ MS 176 clo Mackay School of Mines UNR, Reno, NV 89557,graines@usgs.gov \\ ${ }^{2} 904$ W. Riverside Ave., Room 202, Spokane, WA 99201}

\section{Abstract: Simulation of Rare Spatial-Temporal Events Involving the Interaction of Man and the Environment - Exploration for Mineral Resources}

For public land management in Idaho and western Montana, the U.S. Forest Service (USFS) has requested that the USGS predict where mineral-related activity will occur in the next decade.

Mineral resources of economic value are rare phenomenon. Cellular automata (CA) provide an approach to simulation of this interaction of human activity (the mining industry) and the environment (mineral resources). Any CA modeling the exploration for mineral resources has to account for the following properties. (1) Public land is not uniformly distributed. (2) Exploration permits are only required for public land. (3) Mineral resources constitute the product of rare events that are irregularly distributed. (4) Most mining-industry activity occurs near previous or current exploration activity because of the mature state of knowledge about mineral resources in Idaho and western Montana. The CA implemented is a modified annealed voting rule with spatial and temporal resolution of one $\mathrm{mi}^{2}$ and 1 year calibrated from permits issued by USFS and Bureau of Land Management (BLM) from 1989 to 1998. The modification of the annealed voting rule is that all decisions are probabilistic within constraints based on the spatial distribution of public land and fuzzy/probabilistic information about the existence of mineral resources. The state of the economy and exploration technology is assumed constant for the next decade.

The calibrated CA reproduces the 1989-1998-permit activity with an agreement of 94\%, which increases to $98 \%$ for within one year. Analysis of the confusion matrix and Kappa correlation statistics indicates that the CA underestimates high activity and overestimates low activity. Spatially, the major differences between the actual and calculated activity are that the calculated activity occurs in a slightly larger number of small patches and is slightly more uneven than the actual activity. Using the calibrated CA in a Monte Carlo simulation projecting from 1998 to 2010, an estimate of the probability of mineral activity shows high levels of activity in Boise, Caribou, Elmore, Lincoln, and western Valley counties in Idaho and Beaverhead, Madison, and Stillwater counties in Montana, and generally low activity elsewhere. 
Calibration of this CA is a complex and time-consuming process that requires an automated approach, such as genetic algorithms. Experience gained during the calibration of this CA suggests that the mineral resource information is a critical factor in the quality of the results. With automated calibration, further refinements of how the mineral-resource information is provided to the CA will probably improve the simulation. Because mineral resources are rare events, classical statistical measures of spatial goodness-of-fit of the actual to calculated spatial activity, such as agreement and Spearman's correlation coefficient, are insensitive and misleading. Quantitative measures of goodness-of-fit require further investigation to identify appropriate measures and how to apply these measures in calibration and description of the results. 


\section{Sushil J. Louis ${ }^{1}$ and Gary Raines ${ }^{2}$}

${ }^{I}$ Genetic Algorithm Systems Laboratory, Department of Computer Science, University Of Nevada,Reno(sushil@.cs.unr.edu)

${ }^{2}$ U.S. Geological Survey, MS 176 c/o Mackay School of Mines, University Of Nevada, Reno, Nevada89557(graines@usgs.gov)

\section{Abstract: Predicting Permit Activity with Cellular Automata Calibrated with Genetic Algorithms}

We use a genetic algorithm to calibrate a cellular automaton designed to predict spatially resolved mineral-related activity in Idaho and Western Montana. Any cellular automaton modeling exploration for mineral resources must account for the following properties: (1) Public land is not uniformly distributed, (2) Exploration permits are only required for public land, (3) Mineral resources constitute the product of rare events that are irregularly distributed,and (4) Most mining-industry activity in Idaho and western Montana occurs near previous or current exploration activity. Our automaton simulates activity with spatial and temporal resolution of one $m i^{2}$ and one year with a modified annealed voting rule that is probabilistic and uses information about the existence of mineral resources. The state of the economy and exploration technology is assumed constant for the next decade.

When calibrated, the cellular automaton reproduces the 1989-1998 permit activity with $94 \%$ agreement. Analysis of the confusion matrix and Kappa correlation statistics indicates that the CA underestimates high activity and overestimates low activity. However, calibration of this $\mathrm{CA}$ is a complex and time-consuming process that would benefit from an automated approach. In contrast to the current manual approach, our genetic algorithm calibrator takes a day to calibrate the cellular automaton to fit observed data. This paper describes our genetic algorithm approach to cellular automata calibration and provides further evidence that genetic algorithms are a viable alternative to manual calibration of large cellular automata. Experience gained during the calibration of this CA suggests that the mineral resource information is a critical factor in the quality of the results. With automated calibration, further refinements of how the mineral-resource information is provided to the CA will probably improve the simulation.

Acknowledgement: This work was sponsored by the USGS under a cooperative agreement with the University Of Nevada, Reno. 
Stephen Wolfram, Creator of Mathematica, author of A New Kind of Science, and CEO

of Wolfram Research, Inc

Abstract:

Starting from a few computer experiments, Stephen Wolfram has spent more than twenty years developing a new approach to science, described for the first time in his book A New Kind of Science. Basic to his approach is the idea of studying not traditional mathematical equations but instead rules of the kind embodied in the simplest computer programs. A key discovery is that such rules can lead to behavior that shows immense complexity and mirrors many features seen in nature. Wolfram has built on this to tackle a remarkable array of fundamental problems in science, from the origins of apparent randomness in physical systems, to the development of complexity in biology, the ultimate scope and limitations of mathematics, the possibility of a truly fundamental theory of physics, the interplay between free will and determinism, and the character of intelligence in the universe. When Wolfram's book was released on May 14, 2002, it became an instant bestseller, and is now showing many signs of initiating a major paradigm shift in science. Wolfram's presentation will cover some of the key ideas and discoveries in his book, outlining their implications, and discussing their personal and historical context. An extended question and answer period will be included. 


\section{David H. Wolpert, William G. Macready}

NASA Ames Research Center, MS269-2, Moffett Field, CA, 94035

Bios Group LP, 317 Paseo de Peralta, Santa Fe, NM, 87501

\section{Abstract: Self-Dissimilarity: An Empirically Observable Complexity Measure}

For many systems characterized as "complex/living/intelligent" the spatio-temporal patterns exhibited on different scales differ markedly from one another. For example the biomass distribution of a human body "looks very different" depending on the spatial scale at which one examines that biomass. Conversely, the density patterns at different scales in "dead/simple" systems (e.g., gases, mountains, crystals) do not vary significantly from one another.

Accordingly, we argue that the degrees of self-dissimilarity between the various scales with which a system is examined constitute a complexity "signature" of that system. Such signatures can be empirically measured for many real-world data sets concerning spatio-temporal densities, be they mass densities, species densities, or symbol densities. This allows one to compare the complexity signatures of wholly different kinds of systems (e.g., systems involving information density in a digital computer, vs. species densities in a rain-forest, vs. capital density in an economy, etc). Such signatures can also be clustered, to provide an empirically determined taxonomy of "kinds of systems" that share organizational traits. The precise measure of dissimilarity between scales that we propose is the amount of extra information on one scale beyond that which exists on a different scale. This "added information" is perhaps most naturally determined using a maximum entropy inference of the distribution of patterns at the second scale, based on the provided distribution at the first scale. We briefly discuss using our measure with other inference mechanisms (e.g., Kolmogorov complexity-based inference). 


\section{Bernard P. Zeigler ${ }^{1}$, Mark E. Gettings ${ }^{2}$, Phillip Hammonds ${ }^{3}$ ${ }^{1}$ Arizona Center for Integrative Modeling and Simulation; www.acims.arizona.edu; \\ ${ }^{2}$ U.S. Geological Survey, Tucson, AZ \\ ${ }^{3}$ Northrop-Grumman Information Technology, Ft. Huachuca, AZ}

\section{Abstract: Discrete Events - Multi-resolution Modeling And Simulation of Self-Organizing Criticality In Complex Systems}

As the scale of a simulation modeling effort increases, the acumen required is increasingly difficult to come by. For large enough model size and complexity, human intuition fails and provides little guidance as to what to expect and what constitutes correct behavior. This is especially true of models containing rules whose interaction is not amenable to easy analysis and where phenomena are expected to emerge that cannot be anticipated a priori. When familiarity with the domain is not a guide, generally applicable concepts must be relied upon. That is where the theory of modeling and simulation can be most valuable.

In this paper we first review the general system origins of the theory and framework for modeling and simulation. We continue the background presentation with a brief review of the Discrete Event Systems Specification (DEVS) formalism that expresses the class of discrete event systems within the general class of systems. With this background, we cast concepts of self-organizing criticality (SOC) within the DEVS formalism and multi-resolution modeling within the theory and framework of modeling and simulation. The concepts of morphism and experimental frame make it possible to understand and make rational choices for the tradeoff between the nature of questions addressed, the accuracy of model-supplied answers, and the models' computational efficiency. In particular, we show how the multi-resolution approach of the M\&S framework provides the ability to formulate the appropriate experimental frames for measuring and comparing "activity," a concept that underlies the phenomena of SOC. The framework further inter-relates continuous, discrete time and discrete event models, in both linear and non-linear form, for published earthquake models. We show that DEVS provides the right level of abstraction in that it supports both the appropriate models and the efficient simulation for modeling SOC and other complex systems. 


\section{LIST OF WORKSHOP PARTICIPANTS}

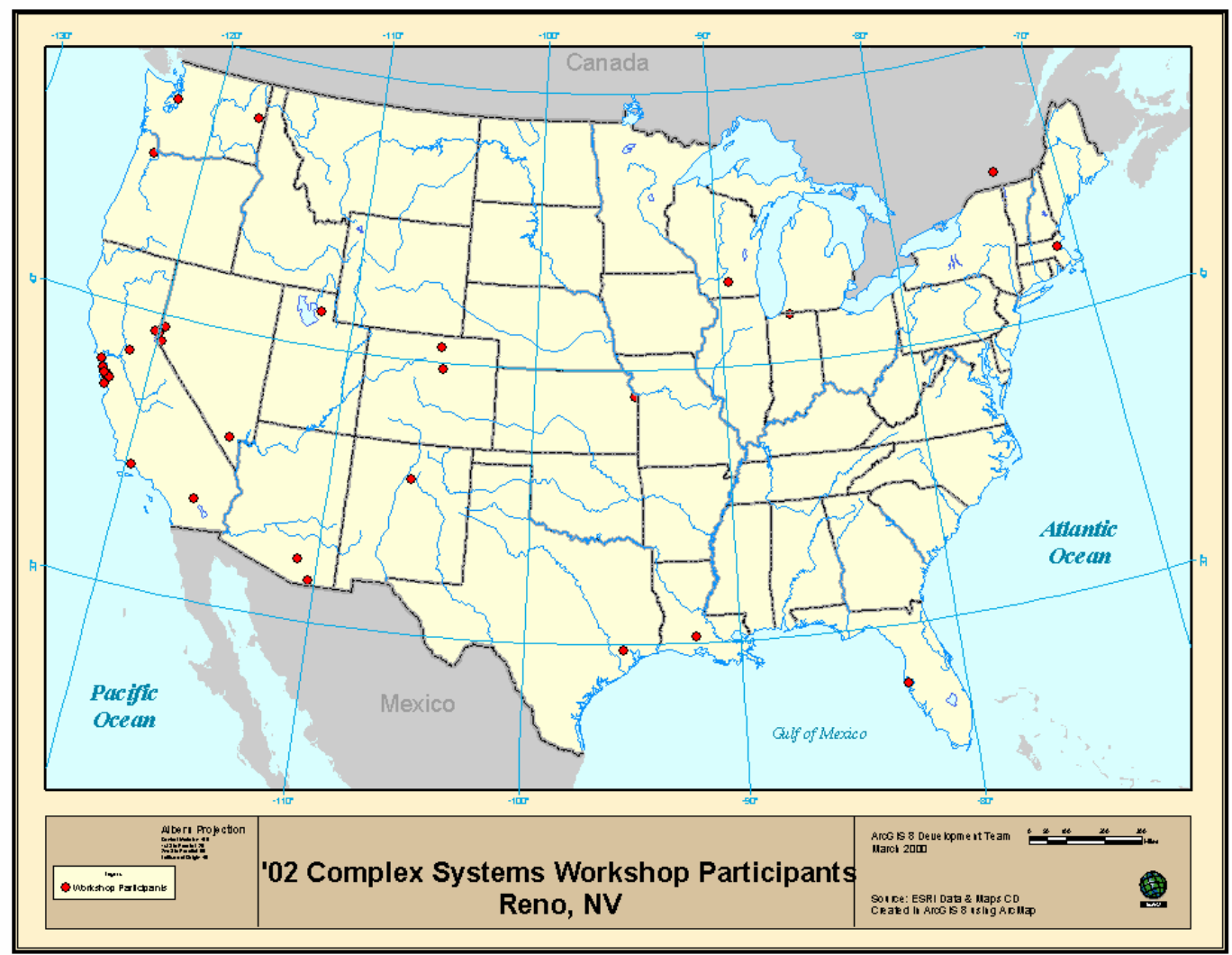

A list of workshop participants is available in Microsoft Excel and FileMaker Pro formats. These data files contain participant's names, research interests, and contact information. Latitude and longitude data are also included to provide compatibility with most Geographic Information Systems software (i.e., ArcView, Geomedia, etc.)

Download the list of participants as an Excel spreadsheet (70 KB)

Download the list of participants as a FileMaker Pro Database file (48 KB) 